\title{
Objektivität im Journalismus neu denken
}

\author{
Journalistische Ethik und Wahrhaftigkeit in Zeiten alternati- \\ ver Fakten
}

Colin Porlezza

\begin{abstract}
"News and truth are not the same thing, and must be clearly distinguished." Walter Lippman, Public Opinion, 1922
\end{abstract}

\section{Einführung}

Am 21. Januar 2017 fand die offizielle Amtseinführung Donald Trumps in Washington, DC statt. In der anschliessenden Pressekonferenz behauptete der damalige Pressesprecher Trumps, Sean Spicer, dass die Feier den grössten je bei einer Amtseinführung registrierten Menschenauflauf aufwies. Die in den Medien gezeigten Fotos vermittelten jedoch ein anderes Bild: Die Vergleiche mit den Aufnahmen von Barack Obamas Amtseinführung zeigten, dass die Zuschauerzahlen bei Trumps Feier deutlich niedriger waren. Bestätigt wurden diese Schlussfolgerungen schliesslich auch durch die Einschätzungen der dafür zuständigen lokalen Parkverwaltung. Trotzdem verkündete die Beraterin Donald Trumps, Kellyanne Conway, tags darauf in einem Interview mit Chuck Todd von der Fernsehsendung Meet the Press, dass es sich bei den Äusserungen Spicers um "alternative Fakten" handelten, wobei sie den Vorwurf der Unwahrheit zurückwies. ${ }^{1}$

Die wiederholten Falschaussagen und Lügen Trumps und seiner Entourage veranlassten zahlreiche etablierte US-Medien, sich mit der Frage zu beschäftigen, wie Journalisten der zunehmenden Vermischung von Wahrheit und Lügen begegnen sollen. Wie sollen Medienschaffende über eine der wichtigsten Nachrichtenquellen berichten, wenn diese kontinuierlich Falschinformationen verbreitet? Damit rückte eine der zentralen ethischen Prinzipien der Profession in den Fokus: die Objektivitätsnorm. Es gibt wenige Aspekte im Journalismus, die häufiger diskutiert wurden, zumal Objektivität

1 Vgl. NBC News, Meet the Press 22.01.2017. Abschrift, https://www.nbcnews. com/meet-the-press/meet-press-01-22-17-n710491 (14.11.2017). 
als zentrale Legitimationsinstanz der journalistischen Berufsethik fungiert. ${ }^{2}$ Die Debatte rund um Objektivität hat deshalb eine lange Tradition. ${ }^{3}$ Vor dem Hintergrund der politischen Umwälzungen im Zusammenhang mit Trump, den Snowden-Enthüllungen oder WikiLeaks hat Objektivität als Diskussionsthema aber deutlich an Brisanz gewonnen.

Die weitreichenden technologischen Veränderungen im medialen Ökosystem ${ }^{4}$ im Zusammenhang mit dem Web führen dazu, dass sich im normativen Fundament des Journalismus Risse bilden. Zwar bleibt Objektivität sowohl im anglo-amerikanischen Raum als auch in Europa immer noch eine zentrale und dominierende Norm, doch der objektive Journalismus verliert an Zuspruch. ${ }^{5}$ Nicht nur, weil der Journalismus seine Bedeutung als Gatekeeper verliert, da vermehrt Nutzer oder Algorithmen Selektionsentscheidungen vornehmen. Er büsst auch an Legitimation ein, da er seine Deutungshoheit innerhalb der Gesellschaft eingebüsst hat. Daraus folgen epistemologische Herausforderungen im Zusammenhang mit der traditionellen Funktion der journalistischen Sinnkonstruktion, ${ }^{6}$ die heute vermehrt von Freunden und Algorithmen übernommen wird.

In der neuen Unübersichtlichkeit eines "high-choice media environments" wird der Journalismus nicht nur mit neuen zeitlichen (schneller!), technischen (konvergenter!), professionellen (partizipativer!) und funktionalen (mehr Kontext und Orientierung!) Anforderungen konfrontiert. ${ }^{7}$ Dem Nutzer erschliesst sich nicht mehr

2 Vgl. Brian McNair, Sociology of Journalism, London 1998, 65.

3 Vgl. Steven Maras, Objectivity in Journalism, Cambridge 2013.

4 Der Begriff des ,neuen medialen Ökosystems“ beschreibt den Umstand, dass der Journalismus die Informationsflüsse in der Gesellschaft nicht mehr länger kontrolliert, sondern stärker als Schnittstelle zwischen vernetzten Nutzern, professionellen Informationsproduzenten und sozialen Medien fungiert. Journalisten übernehmen die Rolle von Mediatoren, welche Informationen filtern, überprüfen und an ihre Publika weiterleiten, um ihnen Orientierung zu bieten. In diesem vernetzten medialen Ökosystem regen sich Nutzer, Journalisten und soziale Medien wiederholt gegenseitig an, sodass sich neue Entwicklungen herausbilden.Vgl. Shayne Bowman/Chris Willis, We Media. How audiences are shaping the future of news and informations, Reston 2003, http://www.hypergene.net/wemedia/download/we_media.pdf (03.01.2018), $12 \mathrm{f}$.

5 Vgl. Frank Esser/Andrea Umbricht, Competing Models of Journalism? Political Affairs Coverage in U.S., British, German, Swiss, French and Italian Newspapers, in: Journalism 15/8, 989-1007.

6 Vgl. Jo Bardoel, Beyond Journalism: A Profession Between Information Society and Civil Society, in: European Journal of Communication 11/3 (1996), 283-302.

7 Vgl. Peter van Aelst u.a., Political Communication in a High-choice Media Environment. A Challenge for Democracy?, in: Annals of the International Communication Association 41/1 (2017), 3-27. 
automatisch, wer in diesem unbegrenzten Informationsangebot die Wahrheit sagt, da das Vertrauen in gesellschaftliche Institutionen schwindet. Dem Journalismus schlägt so vermehrt Misstrauen entgegen, das sich z.B. in Proklamationen wie "Lügenpresse" zeigt.

In Anbetracht der zunehmenden Auflösung der Grenzen zwischen Fakten und Fiktionen, wo sich Wahrheit nur noch schwer fassen lässt, plädiert dieser Beitrag für eine Neudefinition der journalistischen Objektivitätsnorm, basierend auf einem methodischen und pragmatischen Objektivitätsverständnis und den drei Norm-Dimensionen Faktizität, Accountability und Fairness. Damit soll sichergestellt werden, dass sich der Journalismus deutlich von interessengeleiteter Kommunikation wie Öffentlichkeitsarbeit oder Werbung unterscheidet, um die für seine Funktion in der Gesellschaft nötige Autorität zu erhalten. ${ }^{8}$

Der Beitrag geht im Folgenden zunächst auf die Emergenz der Objektivitätsnorm im Journalismus sowie auf epistemologische Fragen der Wissensproduktion im Zusammenhang mit der normativen Verpflichtung des Strebens nach Wahrhaftigkeit ein. Danach beleuchtet der Beitrag den "postmodernen"Wandel im Journalismus, der zu einer Krise des Objektivitätspostulats geführt hat. Zu guter Letzt wird dieser Wandel als Grundlage dafür benützt, ein neues Objektivitäts-Konzept auszuarbeiten, das den Anforderungen des digitalen Journalismus im Zeitalter von Fake News und Propaganda gewachsen ist und der Demokratie auch in Zukunft einen entscheidenden Dienst leisten kann.

\section{Objektivität und Wahrhaftigkeit im Journalismus}

Objektivität als Norm entstand nicht automatisch mit der Entwicklung des Journalismus. Bis spät ins 19. Jahrhundert war der Journalismus in den USA offen parteilich. Frühere Erklärungsversuche führten die Entstehung der Objektivitätsnorm auf ökonomische und technische Gründe zurück. Einerseits habe die Kommerzialisierung der Presse und die daraus hervorgehenden Massenblätter der "Penny Press« dazu geführt, dass sich Journalisten stärker an einer ausgeglichenen Berichterstattung orientierten, mit dem Ziel, eine möglichst grosse Leserschaft zu erreichen. Andererseits sei die Erfindung des Telegraphen verantwortlich dafür, dass sich die journalistische Berichterstattung aufgrund der Störungsanfälligkeit technischer Kom-

8 Vgl. Matt Carlson, Journalistic Authority. Legitimating News in the Digital Era, New York 2017. 
munikationsmittel auf die wesentlichen Fakten beschränkte. ${ }^{9}$ Diese Gründe erklären jedoch nicht, warum Objektivität von Journalisten als verpflichtende Norm für den eigenen Berufsstand akzeptiert wurde, zumal bekannte Verleger wie Randolph Hearst, Joseph Pulitzer oder Arthur Ochs ihre Zeitungen bis spät ins 19. Jahrhundert als Instrument benutzten, um politische Entscheidungsprozesse zu beeinflussen. ${ }^{10}$

Laut Michael Schudson ist die Emergenz der Objektivitätsnorm auf eine generelle Professionalisierung des US-Journalismus zurückzuführen. Dabei spielt die Kommerzialisierung - zusammen mit einer zunehmenden Fakten- und Wissenschaftsorientierung sowie einer generell politkritischen Haltung - eine wichtige Rolle als Voraussetzung für eine Professionalisierung, indem sie Journalisten die Unabhängigkeit von politischen Akteuren wie Parteien ermöglichte. Primär garantiert die Objektivitätsnorm eine stärkere Kohäsion und Kontrolle innerhalb der journalistischen Profession und sorgt gleichzeitig dafür, dass Journalisten im Zuge der gesellschaftlichen Differenzierung ihre eigene Vorstellung von (Wissens-) Produktion - also Fakten sammeln und berichten - einfordern können. ${ }^{11}$ Damit lässt sich die journalistische Tätigkeit von anderen Kommunikationsformen wie Öffentlichkeitsarbeit, Werbung oder Propaganda unterscheiden - was Gaye Tuchman als »strategisches Ritual beschreibt. ${ }^{12}$ Die Objektivitätsnorm soll sicherstellen, dass sich Journalisten an die normative Verpflichtung des Strebens nach Wahrhaftigkeit halten. Es überrascht deshalb nicht, dass sich die $\mathrm{Ob}-$ jektivitätsnorm in den USA im Verlauf der 1920er Jahre durchsetzte, also kurz nach den durch Propaganda gezeichneten Jahren des 1. Weltkriegs. ${ }^{13}$

Diese Auffassung wirft die erkenntnistheoretische Frage auf, inwiefern der Journalismus überhaupt imstande ist, Realität abzubilden. ${ }^{14}$ Die Wissenschaft ist sich hier einig: Er kann dies nicht leisten.

\footnotetext{
9 Vgl. Michael Emery/Edwin Emery, The Press and America. An Interpretive History of the Mass Media, Boston 1996.

${ }^{10} \mathrm{Vgl}$. Michael Schudson, The Objectivity Norm in American Journalism, in: Journalism 2/2 (2001), 149-170.

${ }^{11}$ Vgl. ebd., $152 \mathrm{f}$.

${ }^{12}$ Gaye Tuchman, Objectivity as Strategic Ritual, in: American Journal of Sociology 77/4 (1972), 660-679.

${ }^{13}$ In Europa und anderen Kontinenten verbreitete sich die Objektivitätsnorm hingegen nach dem 2. Weltkrieg. Siehe Svennik Høyer/Horst Pöttker, Diffusion of the News Paradigm 1850-2000, Göteborg 2007.

${ }^{14}$ Siehe dazu Christoph Neuberger, Journalistische Objektivität.Vorschlag für einen pragmatischen Theorierahmen, in: M\&K Medien \& Kommunikationswissenschaft
} 
Journalismus ist eine soziale Konstruktion, d.h. auch Nachrichten werden konstruiert. Dieser Beitrag geht aber von einem moderaten Konstruktivismus aus, der vorsieht, dass vorläufige Aussagen über die Realität durchaus möglich sind, sofern der Erkenntnisprozess und die dazu verwendeten Methoden offengelegt und begründet werden.

Die eigentliche Problematik der Objektivitätsnorm dreht sich jedoch um die epistemologische Frage, ob es möglich und wünschenswert ist, Subjektivität aus der journalistischen Wissensproduktion herauszuhalten: Eine Mehrheit der deutschen Journalisten gibt an, die "Realität genauso ab(zu)bilden, wie sie ist«, wobei sich rund zwei Drittel auch sicher sind, dieses Ziel erreichen zu können. ${ }^{15}$ Dieser Umstand wird dadurch unterstrichen, dass Objektivität bzw. Wahrhaftigkeit in den Ethik-Kodizes von Institutionen der Medienselbstregulierung wie Presseräten explizit als journalistische Normen erwähnt werden. So lautet Ziffer 1 des deutschen Pressekodex (2017) wie folgt: »Die Achtung vor der Wahrheit, die Wahrung der Menschenwürde und die wahrhaftige Unterrichtung der Öffentlichkeit sind oberste Gebote der Presse. ${ }^{16}$

Gleichzeitig zeigt sich, dass Journalisten ein eher diffuses Bild von Objektivität haben und bisweilen auch mehrere Normen unter demselben Konzept subsumieren: Studien wie die von Wolfgang Donsbach und Bettina Klett beschreiben ganze Kriterienkataloge, die Konzepte wie Neutralität, Ausgewogenheit, Sachlichkeit, die Trennung von Nachricht und Meinung, Relevanz, Vollständigkeit, Vielfalt oder Genauigkeit beinhalten. ${ }^{17}$ Ausserdem variiert die Implementation der Objektivitätsnorm je nach Rollenselbstbild. ${ }^{18}$ Insgesamt lässt sich Objektivität deshalb gleichzeitig als normatives Ideal aber auch als Set von journalistischen Praktiken definieren, die sich durch eine Orientierung an Faktizität und Ausgewogenheit auszeichnen. ${ }^{19}$

\footnotetext{
65/2, 406-431.

15 Siegfried Weischenberg/Maja Malik/Armin Scholl, Die Souffleure der Mediengesellschaft, Konstanz 2006, hier 347.

${ }^{16}$ Deutscher Presserat, Pressekodex, http://www.presserat.de/pressekodex/pressekodex/ (14.11.2017).

${ }^{17}$ Vgl. Wolfgang Donsbach/Bettina Klett, Subjective Objectivity. How Journalists in Four Countries Define a Key Term of Their Profession, in: The International Communication Gazette 51/1 (1993), 53-83.

${ }_{18}$ Vgl. Morten Skovsgaard u.a., A Reality Check: How Journalists' Role Perceptions Impact their Implementation of the Objectivity Norm, in: Journalism 14/1 (2013), $22-42$.

${ }^{19}$ Vgl. Schudson, The Objectivity Norm in American Journalism, 149-170.
} 
Objektivität wird als Übereinstimmung der journalistischen Berichterstattung mit überprüfbaren Fakten und Tatsachen verstanden. Die Richtigkeit der Beschreibungen dient in dieser Hinsicht als Operationalisierung der Wahrheit. Darüber hinaus sehen klassische Definitionen der Objektivität vor, dass die Berichterstattung nicht nur akkurat und faktenbasiert, sondern gleichzeitig auch ausgewogen zu sein hat, obwohl der Bezug auf die Ausgewogenheit aufzeigt, wie Objektivität nur im Rahmen der eigenen, subjektiven Wertvorstellung möglich ist. ${ }^{20}$ Das Zusammenspiel der beiden Aspekte Faktizität und Unparteilichkeit ermöglicht nach Ansicht von Brian McNair nicht nur, dass den Aussagen der Journalisten vertraut werden kann, sondern auch, dass Fakten so erst Bedeutung erlangen. ${ }^{21}$ Dadurch lässt sich zwischen Journalist und Nutzer eine Art Vertrag schliessen: "This journalism is objective, therefore it is not contaminated with propaganda or influenced by the subjective, ideological biases and goals of its producers or proprietors. You, the reader, can trust its author to be honest and sincere in his or her account of the complex reality contained within it. $\ll^{22}$

Wie Marcel Broersma aufzeigt, wenden Journalisten diskursive Strategien an, die darauf abzielen, das Publikum von der Wahrhaftigkeit ihrer Darstellungen zu überzeugen. ${ }^{23}$ Indem sie bestimmten Routinen folgen, die sich aus der Objektivitätsnorm ableiten - also die Realität faktenbasiert, neutral, unpersönlich und ausgewogen zu schildern - erscheint der Bericht für die Leser als die Wahrheit. Folglich resultiert die performative Macht des Journalismus nicht eigentlich aus den Inhalten bzw. den Fakten, sondern aus der Art und Weise der Berichterstattung - also den Formaten und Routinen: "The power of media lies not only (and not even primarily) in its power to declare things to be true, but in its power to provide the forms in which the declarations appear. ${ }^{24}$ Allerdings stellt sich die Frage, ob Nutzer dem Journalismus im heutigen News-Ökosystem noch blind vertrauen. In der Schweiz ist das Vertrauen in die Medien

\footnotetext{
${ }^{20} \mathrm{Vgl}$. McNair, The Sociology of Journalism, $68 \mathrm{ff}$.

${ }^{21}$ Brian McNair, After Objectivity. Schudson's Sociology of Journalism in the Era of Post-factuality, in: Journalism Studies 18/10 (2017), 1318-1333, hier 1325.

22 Ebd., 1320. Übersetzung: „Dieser Journalismus ist objektiv und deshalb nicht mit Propaganda oder den subjektiven, ideologischen Verzerrungen der Produzenten kontaminiert. Ihr, die Leser, könnt auf eine ehrliche Beschreibung der komplexen Realität des Autors vertrauen.»

${ }^{23}$ Vgl. Marcel Broersma, The Unbearable Limitations of Journalism. On Press Critique and Journalism's Claim to Truth, in:The International Communication Gazzette $72 / 1$ (2010), 21-33.

${ }^{24}$ Michael Schudson, The Power of News, Cambridge MA 1995, hier 54.
} 
zwar immer noch relativ hoch, aber die News-Avoidance-Strategien der Nutzer zeigen erste Brüche: Rund ein Drittel der befragten Personen geben an, dass sie der journalistischen Berichterstattung nicht mehr trauen. ${ }^{25}$

\section{Die Krise der Objektivitätsnorm}

Die Objektivitätsnorm ist seit jeher umstritten. Häufig wird gerade Objektivität als Ursache für eine verzerrte Berichterstattung angeführt. Lenin prangerte beispielsweise nach der Oktoberrevolution 1917 russische Journalisten an, sie würden einer »bourgeoisen Objektivität " folgen. ${ }^{26}$ Der Revolutionär argumentierte, dass Gesellschaften in unterschiedliche soziale Klassen stratifiziert seien, weshalb es so etwas wie absolute Wahrheit im Journalismus nicht gäbe. Wahrheit würde also immer durch klassenbezogene Wertvorstellungen beeinflusst. Objektivität wird mit der Kritik einer strukturellen Verzerrung konfrontiert, weil Journalisten aufgrund ihrer kulturellen Prädisposition herrschende Eliten als Informationsquellen bevorzugen und Machtstrukturen dadurch zementieren. ${ }^{27}$

Obwohl die Objektivitätsnorm in den USA und Europa weiterhin dominiert, gerät der objektive Journalismus in den letzten Jahren zunehmend unter Druck. Dies dürfte insbesondere auf die grundlegenden Veränderungen im News-Ökosystem zurückzuführen sein. Zum einen hat die Digitalisierung von Kommunikationsmedien dazu geführt, dass sich Öffentlichkeit in kleinere Teilöffentlichkeiten aufspaltet. Damit einher gehen unterschiedliche Kommunikationsbeziehungen sowie vielfältige Interaktionsmodi, die sich nicht mehr auf die klassische Beziehung Sender-Empfänger reduzieren lassen. ${ }^{28}$ Zum anderen bedeutet dies aber auch, dass der klassische Journalismus seine Deutungshoheit innerhalb der Gesellschaft verliert, zumal öffentliche Kommunikation im Rahmen einer Konkurrenzsituation

\footnotetext{
${ }^{25}$ Vgl. Forschungsinstitut Öffentlichkeit und Gesellschaft (fög), Reuters Institute Digital News Report 2017. Ergebnisse für die Schweiz, Zürich 2017, https://www. foeg.uzh.ch/dam/jcr:8d44f1f2-ec81-4cb4-90f0-be453b1bba67/DNR_2017.pdf (14.11.2017).

${ }^{26}$ Brian McNair, Glasnost, Perestroika and the Soviet media, London 1991, hier 18.

${ }^{27}$ Vgl. C. Richard Hofstetter, Bias in the News. Network Television Coverage of the 1972 Election Campaign, Columbus 1976.

${ }^{28}$ Vgl. Otfried Jarren/Ulrike Klinger, Öffentlichkeit und Medien im digitalen Zeitalter: zwischen Differenzierung und Neu-Institutionalisierung, in: Medienkompetenz. Herausforderung für Politik, politische Bildung und Medienbildung, hg. v. Harald Gapski/Monika Oberle/Walter Staufer, Bonn 2017, 33-42.
} 
zu weiteren Akteuren wie Social-Media-Plattformen, Suchmaschinen und nicht-professionellen Bürgerjournalisten stattfindet. Dies wiederum führt dazu, dass der Journalismus nicht nur an Bedeutung, sondern auch an Legitimation und Vertrauen einbüsst, da grundlegende Funktionen wie Nachrichtenselektion, Themensetzung, Orientierung und Nachrichtendistribution von Freunden und Algorithmen übernommen werden.

Dieser "postmoderne Wandel $\aleph^{29}$ führt nicht nur zu einem digitalen Strukturwandel des Journalismus, sondern stellt mit der Objektivität auch einen der ethischen Grundpfeiler der journalistischen Profession infrage. Die damit einhergehenden, epistemologischen Veränderungen lassen sich anhand von vier konkreten Phänomenen gut beobachten.

Erstens stehen Journalisten aufgrund der technologischen Entwicklungen rund um das Web zahlreiche unterschiedliche Formate des Storytellings, Genres und Interaktionsmöglichkeiten zur Verfügung, welche persönliche und informelle Diskurse begünstigen. ${ }^{30}$ Dank der Einführung von Blogs bietet sich Journalisten z.B. die Chance, unabhängig vom engen Korsett objektiver Berichterstattung Themen auf neue und innovative Art zu präsentieren. Im Zuge der Konvergenz lassen sich auch neue Formen des multi- und transmedialen Journalismus realisieren ${ }^{31}$, während auf sozialen Medien wie Facebook oder Twitter der persönliche Austausch mit Nutzern stattfinden kann. Insgesamt zeigt sich, dass Journalisten aufgrund der zahlreichen Darstellungsformen in ihren Diskursen nicht nur subjektiver geworden sind, ${ }^{32}$ sondern dass Emotionen auch im traditionellen Journalismus generell wichtiger werden. ${ }^{33}$

Zweitens führt das Verschwimmen der Grenze zwischen Produzenten und Konsumenten und die dadurch entstehende partizipa-

\footnotetext{
${ }^{29}$ Vgl. Karin Wahl-Jorgensen, Is There a "Postmodern Turn« in Journalism?, in: Rethinking Journalism Again. Societal Role and Public Relevance in a Digital Age, hg. v. Chris Peters/Marcel Broersma, London 2017, 97-111.

${ }^{30}$ Vgl. Melissa Wall, »Blogs of War«. Weblogs as News, in: Journalism 6/2 (2005), 153172.

${ }^{31}$ Vgl. Colin Porlezza/Eleonora Benecchi/Cinzia Colapinto, The Transmedia Revitalization of Investigative Journalism, in: Exploring Transmedia Journalism in the Digital Age, hg. v. Renira Rampazzo Gambarato/Geane Alzamora, Hershey 2018.

${ }^{32}$ Vgl. Rosalind Coward, Speaking Personally. The Rise of Subjective and Confessional Journalism, Basingstoke 2013.

${ }^{33}$ Vgl. Mark Deuze/Charlie Beckett, On the Role of Emotion in the Future of Journalism, in: Social Media + Society 2/3 (2016), 1-6.
} 
tive Kultur ${ }^{34}$ zu Formen der Produsage ${ }^{35}$ wie partizipativem oder Bürger-Journalismus. ${ }^{36}$ Bei dieser Art der Nutzerbeteiligung zeigt sich, dass die Rollen- und Normvorstellungen des klassischen Journalismus häufig nicht übernommen oder sogar abgelehnt werden. Zahlreiche Bürgerjournalisten sprechen traditionellen Journalisten nicht nur ihre Autorität ab, sondern kritisieren sie auch für die an (vermeintlicher) Objektivität ausgerichteten Wissensproduktion. Es zeigt sich, dass Bürgerjournalisten eigenen ethischen Richtlinien folgen, die mit den Ethik-Kodizes traditioneller Institutionen journalistischer Selbstregulierung wie Presseräten nur wenig gemeinsam haben. ${ }^{37}$ Im Gegenteil, Bürgerjournalisten greifen bei ihrer Berichterstattung explizit auf subjektive Formen des Storytellings zurück, um den Standpunkt »einfacher Bürger ${ }^{38}$ wiederzugeben. Diese Entwicklung äussert sich in den epistemologischen Folgen für klassische Journalisten, die sich der Konkurrenz von Bürgerjournalisten ausgesetzt sehen, die frei von einschränkenden professionellen Normen wie Objektivität berichten können und so den Eindruck einer grösseren Authentizität vermitteln. Damit bieten Bürgerjournalisten eine willkommene Alternative zum objektiven und distanzierten "Eunuchen-Journalismus « ${ }^{39}$ etablierter Medienunternehmen. Gerade der Anschein authentischer Berichte vermittelt das Gefühl einer wahrhaftigen, realistischen und unzensierten Berichterstattung, die bei den Nutzern in Zeiten der "Lügenpresse« besonders gut ankommt.

\footnotetext{
${ }^{34}$ Vgl. Harold Jenkins, Fans, Bloggers, and Gamers. Exploring Participatory Culture, New York 2006.

${ }^{35}$ Der Begriff Produsage bedeutet, dass sich im heutigen medialen Ökosystem die Rollen des Konsumenten (consumer) mit derjenigen des (Inhalte-)Produzenten (producer) vermischt haben. Nutzer können Informationen für eigene Projekte verwerten, verändern und neu distribuieren, weshalb sie gleichzeitig auch zu Produzenten werden und deshalb die hybride Rolle des »Produsers« übernehmen. Beispiele für Produsage finden sich im Bürgerjournalismus oder in der Fan-Fiction.

${ }^{36}$ Vgl. Axel Bruns, Gatewatching. Collaborative Online News Production, New York 2005.

${ }^{37}$ Vgl. Michal Kus/Tobias Eberwein/Sergio Splendore/Colin Porlezza, Training or Improvisation? Citizen Journalism and their Educational Backgrounds - A comparative View, in: Journalism Practice 11/2-3 (2017), 355-372.

${ }^{38} \mathrm{Vgl}$. Stuart Allan, Citizen Witnessing. Revisioning Journalism in Times of Crisis, New York 2013.

${ }^{39}$ Peter Blunschi, Nach der Anti-DSI-Kampagne: Wie "neutral« soll politischer Journalismus sein?, http://www.watson.ch/Schweiz/Kommentar/721486812-Nach-derAnti-DSI-Kampagne--Wie-\%C2\%ABneutral\%C2\%BB-soll-politischer-Journalismus-sein- (18.10.2017).
} 
Drittens gerät die Objektivitätsnorm durch die zunehmend vernetzte und über die Grenzen der Redaktion hinausgehende journalistische Produktion unter Druck. In spezifischen Bereichen wie dem Datenjournalismus arbeiten Journalisten zunehmend mit Vertretern anderer Professionen wie Hacker, Programmierer oder Visualisierungsexperten zusammen, die über unterschiedliche professionelle Kulturen verfügen und denen die journalistische Professionsethik fremd ist. Häufig sind (Daten-)Journalisten beim Sammeln von Daten auf die Zusammenarbeit mit anderen Organisationen oder NGOs angewiesen, die ihre eigene Agenda haben. Ähnliche Phänomene zeigen sich auch im investigativen Journalismus: In der Zusammenarbeit mit Whistleblowing-Plattformen oder Whistleblowern wie Edward Snowden ist der Übergang vom Journalist zum Aktivist zunehmend fliessend. So lehnt beispielsweise der amerikanische Journalist Glenn Greenwald, der im Zuge der Snowden-Leaks und der Überwachungsskandale Berühmtheit erlangte, die Dichotomie zwischen Journalist und Aktivist klar ab, da er Journalismus auch als eine Form von Aktivismus im Dienste der Demokratie sieht: «I think all journalism is a form of activism, but not all activism is journalism.» ${ }^{40}$

Als vierten und letzten Punkt hat die Digitalisierung des Journalismus zu einer Bedeutungszunahme der Transparenz als Instrument der Qualitätssicherung geführt. Diese spielt nicht nur als Legitimationsstrategie für einen offenen Journalismus eine zentrale Rolle, indem Journalisten im Netz ihre Recherchemethoden ausführen und begründen, auf ihre Quellen verweisen, Fehler korrigieren oder ihre Daten für die weitere Verwendung zur Verfügung stellen können. Transparenz spielt auch im Hinblick auf die Offenlegung von Interessen eine zentrale Rolle. Sie ist deshalb nicht nur als ethische Handlungsanweisung bedeutungsvoll, sondern kann auch zu mehr Vertrauen in die Leistungen des Journalismus beitragen. ${ }^{41}$ Daraus resultieren Veränderungen, die sich nicht nur in einer zunehmenden Rechenschaftspflicht gegenüber dem Publikum zeigen, sondern auch in einer veränderten Verantwortungskultur, die sich nicht mehr ausschliesslich auf die Rolle des Gatekeepers beschränkt. Es geht um die Entwicklung einer neuen Beziehungsethik. ${ }^{42}$ Dabei wird

\footnotetext{
${ }^{40}$ Glenn Greenwald, Tweet vom 28.12.2013, https://twitter.com/ggreenwald/status/416869471752429569 (18.10.2017).

${ }^{41} \mathrm{Vgl}$. Andreas Funck, Die transparente Redaktion. Ein Ansatz für die strategische Öffentlichkeitsarbeit von Zeitungsverlagen, Wiesbaden 2016.

${ }^{42}$ Jane Singer, Norms and the Network. Journalistic Ethics in a Shared Media Space, in: Journalism Ethics. A Philosophical Approach, hg. v. Christopher Myers, Oxford 2010, 117-129.
} 
das traditionelle journalistische Rollenselbstverständnis des neutralen Vermittlers insbesondere durch eine erhöhte Bereitschaft zum Dialog und zur Interaktion mit dem Nutzer auf die Probe gestellt. Ein solches Leitbild bedeutet nicht nur konkrete Änderungen im Hinblick auf die journalistische Praktik, sondern auch ein neues Verständnis traditioneller ethischer Standards.

Ereignisse wie Occupy Wall Street ab 2011 haben konkret aufgezeigt, dass die Grenzen zwischen professionellem Journalismus und Bürgerjournalismus, zwischen Objektivität und Subjektivität, zwischen Berichterstattung und Aktivismus zusehends verschwimmen. Der technologische Wandel und seine Konsequenzen erschüttern den Journalismus in seinen normativen Grundfesten, was sich in professionsethischen Umbrüchen wie einer zunehmenden Unschärfe des Objektivitätspostulats - und deshalb auch der Wahrhaftigkeit des Journalismus - äussert.

\section{Zwischen pragmatischer Objektivität und Accoun- tability}

Bereits im ersten journalistischen Handbuch von 1695 wies Kaspar Stieler darauf hin, dass Zeitungen ihre Reputation nur aufgrund der Wahrhaftigkeit ihrer Berichte entfalten. ${ }^{43}$ Diese Verpflichtung der Journalisten gegenüber der Wahrheit hat sich in den letzten 300 Jahren nicht verändert. Auch heute noch stellen Journalisten Wahrhaftigkeit als Fundament der journalistischen Berichterstattung dar, wobei das Publikum diese von den Journalisten geförderte Erwartung weitgehend übernommen hat. Allerdings fällt es dem Journalismus zunehmend schwer, die Mängel seiner eigenen Routinen und normativen Ausrichtung zu kaschieren. Immer wieder kommt es zu Vorfällen, die der Glaubwürdigkeit des Journalismus zusetzen: Fälschungsskandale, Instrumentalisierungen durch PR-Apparate von Wirtschaftsunternehmen oder der Politik, Phänomene wie Lügenpresse oder Fake News, und nicht zuletzt auch die mangelnde Finanzierung der Institution Journalismus setzen zentrale berufsethischen Grundsätze des Journalismus unter Druck. Gerade in den USA zeigt sich diese Entwicklung an sinkenden Glaubwürdigkeitswerten der Medien.

Wie kann der Journalismus im gegenwärtigen Medienumbruch auf solche Herausforderungen reagieren? Und wie lassen sich

${ }_{43}$ Vgl. Kaspar Stieler, Zeitungs Lust und Nutz, hg. v. Gert Hagelweide, Bremen 1969. 
grundsätzliche epistemologische Veränderungen im Kontext neuer Phänomene wie Produsage, Bürgerjournalismus, Big Data und vernetzter Produktion sinnvoll in einen neuen, beziehungsethischen Rahmen integrieren? Ein erster Schritt hin zu einer ethischen Neuausrichtung besteht darin, Objektivität nicht mehr als fixes und verbindliches normatives Konzept zu verstehen (was es für Journalisten aufgrund der zahlreichen zugeschriebenen Definitionen sowieso nicht ist), sondern als Prozess. Dabei geht es nicht um einen strategischen Diskurs, dessen Ziel darin besteht, das Publikum trotz aller Defizite von der Wahrhaftigkeit seiner Berichte zu überzeugen. Vielmehr geht es darum, dass Journalisten die Methoden ihrer Arbeit offenlegen, sodass der Erkenntnisprozess transparent gemacht wird, ähnlich wie dies in der Wissenschaft der Fall ist. ${ }^{44}$

Dieses Verständnis fusst auf den Postulaten des »kritischen Rationalismus". Sie besagen, dass Erkenntnis in jedem Fall vorläufig ist, die Beschreibung der Realität aber auf der Basis einer sorgfältigen Prüfung erfolgt, welche die Richtigkeit der Fakten und Erklärungen sicherstellen soll. Allerdings kann sich die Situation - und deshalb auch deren Beschreibung - aufgrund neuer Erkenntnisse jederzeit wieder ändern. Objektivität - und mit ihr die Wahrhaftigkeit des journalistischen Diskurses - wird erst über die Offenlegung und Beschreibung der (Defizite der) eigenen Methoden derVerifikation, Wissensproduktion und Sinnkonstruktion etabliert. Dies bedeutet jedoch nicht, dass »Transparenz die neue Objektivität« ist - wie Bürgerjournalisten oftmals ihre Leitmaxime beschreiben. Der journalistische Anspruch auf Wahrheit und die Objektivitätsnorm repräsentieren zentrale Aspekte, wenn es um die Kontrollfunktion des Journalismus in der Gesellschaft geht. ${ }^{45}$ Allerdings setzt diese alternative Auffassung von Objektivität eine journalistische Selbstreflexion voraus, die beinhaltet, dass Journalisten sich kritisch mit ihren Routinen, den Grenzen ihrer Performanz, aber auch Fragen ihrer Verantwortung im Kontext neuer Kommunikationsmöglichkeiten und Interaktionsmodi auseinandersetzen.

Der durch die Digitalisierung hervorgerufene Medienumbruch hat zu neuen und ungeklärten ethischen Herausforderungen geführt, die sich in einem relationalen Ethikverständnis niederschlagen. Aus diesem Grund muss sich der Objektivitätsbegriff auch mit der

\footnotetext{
${ }^{44}$ Vgl. Senja Post, Scientific Objectivity in Journalism? How Journalists and Academics Define Objectivity, Assess its Attainability, and Rate its Desirability, in: Journalism 16/6 (2015), 730-749.

${ }^{45}$ Vgl. Stephen Ward, Invention of Journalism Ethics. The Path to Objectivity and Beyond, Montreal 2004.
} 
Rechenschaftspflicht des Journalismus gegenüber seinem Publikum befassen. Der digitale Journalismus verfügt im Netz über zahlreiche Möglichkeiten und Instrumente, wie er gegenüber den Nutzern Rechenschaft für seine (Fehl-)Leistungen ablegen, aber auch Themen wie Objektivität und Wahrhaftigkeit öffentlich diskutieren kann. In einer durch Partizipation geprägten Gesellschaft ist es nicht nur sinnvoll sondern notwendig, dass Journalisten einerseits selbständig über ihre Leistungen und Verfehlungen reflektieren, andererseits aber auch den Dialog mit ihren zahlreichen Stakeholder-Gruppen pflegen. In Zeiten von Fake-News-Ökosystemen und Lügenpresse dürfte eine öffentliche Auseinandersetzung über den Journalismus dessen Autorität nicht untergraben, sondern eher stärken.

Damit ist aber die Frage nach dem Umgang mit walternativen Fakten" noch nicht beantwortet. Wie sollen Journalisten mit Fakten verdrehenden Präsidenten oder Klimawandel-Leugnern umgehen? Traditionelle Auffassungen von Objektivität, mit ihrer starken Ausrichtung auf die Ausgeglichenheit zwischen den Positionen, fördert nicht nur die episodische orientierte Berichterstattung, die Ursachen und Wirkungszusammenhänge ausblendet, sondern führt vor allem zu verzerrenden Nullsummenspielen zwischen vermeintlich gleichgestellten Akteuren oder Positionen. ${ }^{46}$ Der Journalismus tut sich mit solchen Fragen immer noch schwer, zumal er in der Politik-, Krisen-, Kriegs- und Umweltberichterstattung kontinuierlich mit gegensätzlichen Positionen konfrontiert wird, die es gegeneinander abzuwägen gilt. Dieser Beitrag plädiert dafür, dass Objektivität - wie dies Ward (2017) zutreffend umschreibt - pragmatisch aufgefasst wird, indem der Journalist auf der Grundlage von verifizierten Fakten eine nach bestem Wissen und Gewissen transparente und faire Einschätzung vornimmt und diese durch die präzise Beschreibung der Ereignisse und den jeweiligen Argumenten begründet. ${ }^{47}$

Dieses Vorgehen steht zwar nicht im Einklang mit dem Prinzip der Neutralität in der Berichterstattung. Allerdings hat sich gezeigt, dass Nutzer diese Art der Berichterstattung durchaus schätzen. Das dürfte daran liegen, dass der Journalismus seiner Orientierungsfunktion nachkommt, indem er Fakten eben nicht nur verifiziert, sondern auch abwägt und einordnet. Dies erfordert zwar eine besondere

${ }^{46} \mathrm{Vgl}$. Sandrine Boudana, Impartiality is not Fair: Towards an Alternative Approach to the Evaluation of Content Bias in News Stories, in: Journalism 17/5 (2016), 600618.

${ }^{47}$ Vgl. Stephen Ward, Radical Ethics in a Time of Trump. How to Practice Democratically Engaged Journalism, http://mediashift.org/2017/03/radical-ethics-timetrump-democratically-engaged-journalism/ (19.10.2017). 
professionelle Sorgfalt, bietet in einer durch Unübersichtlichkeit gekennzeichneten Öffentlichkeit aber zusätzliche Hilfe bei der Einschätzung von Ereignissen oder Aussagen. Eine solche Auffassung von Objektivität erlaubt es Journalisten, "to make explicit evaluations and judgments, so long as such interpretations are grounded in fact, logic and other objective tests. ${ }^{48}$

Es braucht ein neues Konzept von Objektivität im Journalismus, das auf Faktizität, Rechenschaftspflicht und Fairness aufbaut. Journalisten müssen sich dabei nicht zwischen der Rolle des objektiven Journalisten und der des Aktivisten entscheiden. Es geht darum, Orientierung in einer unübersichtlichen digitalen Medienwelt anzubieten. Dafür müssen sich Journalisten in Zeiten von Trump und Fake News aber mit den grundsätzlichen ethischen Prinzipien ihrer Profession auseinandersetzen und entscheiden, welche Rolle sie in der Gesellschaft und der Demokratie weiterhin spielen wollen. Nur wenn sich der Journalismus den Herausforderungen der digitalen Öffentlichkeit stellt, wird er weiterhin seiner zentralen Bedeutung in und für die Demokratie gerecht. Einschätzungen auf der Basis von Fakten anzubieten ist der erste Schritt, um die verlorene Autorität wieder zurückzugewinnen: "Wer es unternimmt zu sagen, was ist legei ta eonta-, kann nicht umhin, eine Geschichte zu erzählen, und in dieser Geschichte verlieren die Fakten bereits ihre ursprüngliche Beliebigkeit und erlangen eine Bedeutung, die menschlich sinnvoll ist. « ${ }^{49}$ Darauf müssen sich Journalisten aber zuerst einlassen.

- Colin Porlezza ist Oberassistent am Institut für Publizistikwissenschaft und Medienforschung der Universität Zürich. Zu seinen Arbeitsgebieten gehören Datenjournalismus, Media Accountability und Transparenz im Journalismus.

\footnotetext{
${ }^{48}$ Stephen Ward, Pragmatic News Objectivity. Objectivity with a Human Face, Cambridge MA 1999, hier 8 .

${ }^{49}$ Hannah Arendt, Wahrheit und Politik [dt. 1969], in: Dies., Wahrheit und Lüge in der Politik. Zwei Essays, München/Zürich 2013, 44-92, hier 89.
} 